\title{
MICROWAVE ASSISTED SYNTHESIS OF $N$-(1-ISOPROPYL-2- OXOINDOLIN-5-YL) SUBSTITUTED AMIDE DERIVATIVES AND THEIR ANTIMICROBIAL ACTIVITY
}

\author{
P. Shankaraiah ${ }^{1, *}$, V. Sunitha ${ }^{2}$, A. Kishore Kumar' ${ }^{1}$ and R. Madhu ${ }^{2}$ \\ ${ }^{1}$ Department of Humanities and Sciences, Vardhaman College of Engineering, Shamshabad, \\ Hyderabad-501 218, Telangana, India \\ ${ }^{2}$ Department of Chemistry, University College of Science, Osmania University, Saifabad, \\ Hyderabad-500004, Telangana, India \\ E-mail: shankarpou@gmail.com
}

\begin{abstract}
A series of $\mathrm{N}$-(1-isopropyl-2-oxoindolin-5-yl)substituted amide derivatives 6a-h has been synthesized with 5amino-1-isopropylindolin-2-one using HATU and EDC. $\mathrm{HCl}$ under microwave conditions. All final compounds were characterized by different spectral techniques. These target compounds $(\mathbf{6} \mathbf{a}-\mathbf{h})$ were assessed for their antimicrobial activity and showed significant activity.Among the compounds tested $\mathbf{6 f}, \mathbf{6 h}, \mathbf{6 e}, \mathbf{6 g}$ and $\mathbf{6 b}$ were found potent antimicrobial activities at concentrations 75 and $100 \mu \mathrm{g} / \mathrm{mL}$.
\end{abstract}

Keywords: Benzimidazole, Heterocyclic, HATU, EDC.HCl.

(C) RASĀYAN. All rights reserved

\section{INTRODUCTION}

In the continuous development of new drugs, some of the heterocyclic compounds have been found important source for development of new bioactive compounds. Among these, indolin-2-one derivatives possess a vital role such as antimicrobial, ${ }^{1}$ anticancer, ${ }^{2}$ antiproliferative ${ }^{3}$ and anti-inflammatory activities. ${ }^{4}$ The increasing significance in biological profile of indolin-2-one ${ }^{5-10}$ derivatives prompted us to the synthesis of new heterocyclic compounds which are pharmacologically active.

In recent years, radiation like microwaves (MWs) has been used to establish the reaction conditions for number of organic reactions. ${ }^{11} \mathrm{MW}$ technique provides simple, efficient, economical and safe mimicking to green chemistry. Reactions executed under MW condition ensure fast and good in yields than conventional heating. This irradiation method is alternative to the conventional method.

Inspired by above mentioned biological activities and the advantages of microwave irradiation over conventional method, herein we report the synthesis of the amide derivatives of indolin-2-one(6a-h) from 5-amino-1-isopropylindolin-2-one (4) and different acids (5a-g) under microwave conditions in high yields. These derivatives were screened for their antimicrobial activity against eight bacterial and three fungal species.

\section{EXPERIMENTAL}

Bruker AV-400 NMR spectrometer used for NMR spectra forCDCl ${ }_{3}$ solutions. The chemical shifts were measured in ppm against internal TMS. ESI mass spectra were recorded on a QSTARXL hybrid MS system. TLC was carried out on Merck silica gel $60 \mathrm{~F}_{254}$ TLC plates.

\section{General Procedure for the Synthesis of Compounds 6a-h \\ Conventional Heating Method}

To a stirred individual solutions of 5-amino-1-isopropylindolin-2-one (4) (0.2 g, 1.05mmol), substituted acids $(\mathbf{5 a}-\mathbf{h})(1.05 \mathrm{mmol})$ in DMF $(10 \mathrm{~mL})$ was added EDC.HCl $(1 \mathrm{mmol})$, HATU $(1.2 \mathrm{mmol})$ and the reaction mixture was stirred for $12 \mathrm{hat} 80{ }^{\circ} \mathrm{C}$. After complete conversion of the 4into products as indicated

Rasayan J. Chem., 12(4), 1850-1856(2019)

http://dx.doi.org/10.31788/RJC.2019.1245445

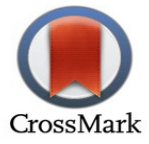




\section{RASĀYAN J. Chem.}

Vol. 12 | No. 4 |1850 - 1856| October - December | 2019

by the TLC the reaction mixture was poured over crushed ice, solid obtained was filtered, washed with excess of water and dried. The crude product obtained was purifiedwith100-200silicameshwith $30 \%$ EtOAc/hexaneas eluent.

\section{Microwave Irradiation Method}

To individual solutions of 5-amino-1-isopropylindolin-2-one (4) $(0.2 \mathrm{~g}, 1.05 \mathrm{mmol})$, substituted acids $(\mathbf{5 a - h})(1.05 \mathrm{mmol})$ in dimethylformamide $(10 \mathrm{~mL})$ was added EDC.HCl $(1 \mathrm{mmol})$, HATU $(1.2 \mathrm{mmol})$ and the mixture was employed in a microwave tube and exposed to microwave irradiation at $180 \mathrm{~W}$ for 8-20 min. After consumption of the compound 4 into products. The reaction mixture was poured over crushed ice, solid obtained was filtered. The crude product obtained was purified with column chromatography using 100-200 silica mesh eluting with 30\%EtOAc/hexane.

\section{2-Iodo-N-(1-isopropyl-2-oxoindolin-5-yl)nicotinamide (6a)}

Brown solid; M.P. 168-170 ${ }^{\circ} \mathrm{C} ;{ }^{1} \mathrm{H}$ NMR $\left(400 \mathrm{MHz}, \mathrm{CDCl}_{3}\right) \delta$, ppm $1.46(\mathrm{~d}, J=4.72 \mathrm{~Hz}, 6 \mathrm{H}), 3.54$ (s, $2 \mathrm{H}), 4.68-4.67(\mathrm{~m}, J=1 \mathrm{H}), 7.01(\mathrm{~d}, J=8.48 \mathrm{~Hz}, 1 \mathrm{H}), 7.50-7.38(\mathrm{~m}, 2 \mathrm{H}), 7.67(\mathrm{~s}, 1 \mathrm{H}), 8.39(\mathrm{~d}, J=4.49$ $\mathrm{Hz}, 1 \mathrm{H}), 8.50(\mathrm{~d}, J=15.26 \mathrm{~Hz}, 1 \mathrm{H}), 8.67$ (ddd, $J=9.75,7.64,1.82 \mathrm{~Hz}, 1 \mathrm{H}) .{ }^{13} \mathrm{C}$ NMR $(100 \mathrm{MHz}$, $\left.\mathrm{CDCl}_{3}\right) \delta$, ppm174.4, 159.7, 150.6, 143.5, 141.0, 131.5, 125.8, 122.7, 122.6, 120.3, 118.4, 116.4, 166.2, 109.7, 43.6, 36.0, 19.2.Anal. Calcd for: $\mathrm{C}_{17} \mathrm{H}_{16} \mathrm{IN}_{3} \mathrm{O}_{2}: \mathrm{C}, 48.47 ; \mathrm{H}, 3.83 ; \mathrm{I}, 30.13 ; \mathrm{N}, 9.98 ;$ O, 7.60. Found: C, 48.41; H, 3.82; N, 9.96.<smiles>O=C1Nc2ccccc2C1=O</smiles><smiles>CC(C)I</smiles>
$0^{\circ} \mathrm{C}$ to $\mathrm{rt}, 10 \mathrm{~min}$<smiles>CC(C)N1C(=O)C(=O)c2ccccc21</smiles>$$
\text { . }
$$<smiles>[H]</smiles><smiles>[R]C(=O)Nc1ccc2c(c1)CC(=O)N2C(C)C</smiles><smiles>CCCOC(=O)N1C(=NN=N)Cc2cc(N)ccc21</smiles><smiles>CC(C)N1C(=O)Cc2cc([N+](=O)[O-])ccc21</smiles><smiles>[R]=CCc1cccnc1I</smiles>

$5 a$<smiles>CCc1ccc(C)cc1</smiles>

$5 e$<smiles>Cc1cccnc1Br</smiles>

$5 b$<smiles>CCc1ccc(S(C)(=O)=O)cc1</smiles>

$5 f$<smiles>Cc1cccc(I)n1</smiles>

5c<smiles>CCc1ccnn1C</smiles>

$5 \mathrm{~g}$<smiles>Cc1cccc(Br)n1</smiles>

$5 d$<smiles>Cc1conc1C</smiles>

$5 \mathrm{~h}$

Scheme-1: Synthesis of N-(1-isopropyl-2-oxoindolin-5-yl) Substituted Amide Derivatives 
RASĀYAN J. Chem.

Vol. 12 | No. 4 |1850 - 1856| October - December | 2019

2-Bromo-N-(1-isopropyl-2-oxoindolin-5-yl)nicotinamide(6b)

White solid; M.P. $158-159{ }^{\circ} \mathrm{C} ;{ }^{1} \mathrm{H}$ NMR $\left(400 \mathrm{MHz}, \mathrm{CDCl}_{3}\right) \delta$, ppm $1.48(\mathrm{~d}, J=4.32 \mathrm{~Hz}, 6 \mathrm{H}), 3.58$ (s, $2 \mathrm{H}), 4.66-4.56(\mathrm{~m}, J=1 \mathrm{H}), 7.03(\mathrm{~d}, J=8.42 \mathrm{~Hz}, 1 \mathrm{H}), 7.56-7.33(\mathrm{~m}, 2 \mathrm{H}), 7.67(\mathrm{~s}, 1 \mathrm{H}), 8.36(\mathrm{~d}, J=4.50$ $\mathrm{Hz}, 1 \mathrm{H}), 8.48(\mathrm{~d}, J=15.11 \mathrm{~Hz}, 1 \mathrm{H}), 8.62(\mathrm{ddd}, J=9.73,7.65,1.84 \mathrm{~Hz}, 1 \mathrm{H}) .{ }^{13} \mathrm{C} \mathrm{NMR}(100 \mathrm{MHz}$, $\left.\mathrm{CDCl}_{3}\right) \delta$, ppm 174.3, 164.2, 151.2, 142.4, 141.1, 132.2, 123.4, 122.4, 122.6, 121.3, 118.8, 116.2, 108.7, 43.5, 35.9, 19.1. Anal.Calcd. for: $\mathrm{C}_{17} \mathrm{H}_{16} \mathrm{BrN}_{3} \mathrm{O}_{2}$ : C, 54.56; H, 4.31; Br, 21.35; N, 11.23; O, 8.55. Found: C, 48.41; H, 4.30; N, 11.21.

\section{6-Iodo-N-(1-isopropyl-2-oxoindolin-5-yl)picolinamide(6c)}

Brownish solid; M.P. 171-173 ${ }^{\circ} \mathrm{C}$; ${ }^{1} \mathrm{H}$ NMR $\left(400 \mathrm{MHz}, \mathrm{CDCl}_{3}\right) \delta$, ppm 1.51-1.46 (m, 6H), 3.54 (s, 2H), $4.68(\mathrm{~d}, J=7.02 \mathrm{~Hz}, 1 \mathrm{H}), 7.01(\mathrm{~d}, J=8.53 \mathrm{~Hz}, 1 \mathrm{H}), 7.54(\mathrm{dd}, J=8.47,2.01 \mathrm{~Hz}, 1 \mathrm{H}), 7.67(\mathrm{~d}, J=7.94$ $\mathrm{Hz}, 1 \mathrm{H}), 7.75(\mathrm{t}, J=7.73 \mathrm{~Hz}, 2 \mathrm{H}), 8.24(\mathrm{~d}, J=7.53 \mathrm{~Hz}, 1 \mathrm{H}), 9.56(\mathrm{~s}, 1 \mathrm{H}) .{ }^{13} \mathrm{C} \mathrm{NMR}(100 \mathrm{MHz}$, $\left.\mathrm{CDCl}_{3}\right) \delta$, ppm 173.49, 162.1, 151.36, 140.26, 131.00, 124.96, 121.36, 119.50, 117.28, 110.00, 77.33, 77.01, 76.31, 43.56, 36.21, 19.35.Anal. Calcd for: $\mathrm{C}_{17} \mathrm{H}_{16} \mathrm{IN}_{3} \mathrm{O}_{2}: \mathrm{C}, 48.47 ; \mathrm{H}, 3.83 ; \mathrm{I}, 30.13$; N, 9.98; O, 7.60. Found: C, 48.46; H, 3.81; N, 9.97.

\section{6-Bromo-N-(1-isopropyl-2-oxoindolin-5-yl)picolinamide(6d)}

White solid; M.P. $198-200{ }^{\circ} \mathrm{C} ;{ }^{1} \mathrm{H}$ NMR $\left(400 \mathrm{MHz}, \mathrm{CDCl}_{3}\right) \delta$, ppm 1.51-1.46 (m, 6H), 3.54 (s, $\left.2 \mathrm{H}\right), 4.68$ $(\mathrm{d}, J=7.04 \mathrm{~Hz}, 1 \mathrm{H}), 7.01(\mathrm{~d}, J=8.51 \mathrm{~Hz}, 1 \mathrm{H}), 7.56(\mathrm{dd}, J=8.49,2.02 \mathrm{~Hz}, 1 \mathrm{H}), 7.67(\mathrm{~d}, J=7.93 \mathrm{~Hz}$, $1 \mathrm{H}), 7.79 \mathrm{t}(J=7.75 \mathrm{~Hz}, 2 \mathrm{H}), 8.26(\mathrm{~d}, J=7.55 \mathrm{~Hz}, 1 \mathrm{H}), 9.64(\mathrm{~s}, 1 \mathrm{H}) .{ }^{13} \mathrm{C} \mathrm{NMR}\left(100 \mathrm{MHz}, \mathrm{CDCl}_{3}\right) \delta$, ppm 174.50, 163.2, 150.47, 141.4, 139.97, 130.98, 125.86, 121.48, 119.48, 117.49, 109.84, 77.35, 77.03, 76.71, 43.66, 36.21, 19.35.Anal. Calcd for: $\mathrm{C}_{17} \mathrm{H}_{16} \mathrm{BrN}_{3} \mathrm{O}_{2}$ : C, 54.56; H, 4.31; Br, 21.35; N, 11.23; O, 8.55. Found: C, $54.51 ; \mathrm{H}, 4.29 ; \mathrm{N}, 11.21$.

\section{N-(1-Isopropyl-2-oxoindolin-5-yl)-2-(4-(trifluoromethyl)phenyl)acetamide(6e)}

White solid; M.P. $162-163{ }^{\circ} \mathrm{C} ;{ }^{1} \mathrm{H}$ NMR $\left(400 \mathrm{MHz}, \mathrm{CDCl}_{3}\right) \delta$, ppm $1.46(\mathrm{~d}, J=10.88 \mathrm{~Hz}, 6 \mathrm{H}), 3.44$ (s, $2 \mathrm{H}), 3.74(\mathrm{~d}, J=21.89 \mathrm{~Hz}, 2 \mathrm{H}), 4.63(\mathrm{~d}, J=6.94 \mathrm{~Hz}, 1 \mathrm{H}), 6.90(\mathrm{~d}, J=8.45 \mathrm{~Hz}, 1 \mathrm{H}), 7.26-7.17(\mathrm{~m}, 1 \mathrm{H})$, $7.45(\mathrm{dd}, J=15.04,10.42 \mathrm{~Hz}, 4 \mathrm{H}), 7.62(\mathrm{~d}, J=7.88 \mathrm{~Hz}, 2 \mathrm{H}) .{ }^{13} \mathrm{C} \mathrm{NMR}\left(100 \mathrm{MHz}, \mathrm{CDCl}_{3}\right) \delta, \mathrm{ppm}$ 174.52, 168.31, 140.59, 138.68, 132.09, 129.69, 125.86, 125.73, 119.80, 117.95, 109.73, 77.36, 77.04, 76.73, 44.06, 43.67, 36.12, 19.28.Anal. Calcd for: $\mathrm{C}_{20} \mathrm{H}_{19} \mathrm{~F}_{3} \mathrm{~N}_{2} \mathrm{O}_{2}$ : C, 63.82; H, 5.09; F, 15.14; N, 7.44; O, 8.50. Found: C, 63.80; H, 5.08; N, 7.43.

\section{N-(1-Isopropyl-2-oxoindolin-5-yl)-2-(4-(methylsulfonyl)phenyl)acetamide(6f)}

White solid; M.P. 121-123 ${ }^{\circ} \mathrm{C}$; ${ }^{1} \mathrm{H}$ NMR $\left(400 \mathrm{MHz}, \mathrm{CDCl}_{3}\right) \delta$, ppm $1.45(\mathrm{~d}, J=2.87 \mathrm{~Hz}, 6 \mathrm{H}), 3.07$ (s, $3 \mathrm{H}), 4.79-4.49(\mathrm{~m}, 1 \mathrm{H}), 3.81(\mathrm{~s}, 2 \mathrm{H}), 3.46(\mathrm{~s}, 2 \mathrm{H}), 7.03-6.82(\mathrm{~m}, 1 \mathrm{H}), 7.27(\mathrm{~s}, 2 \mathrm{H}), 7.56(\mathrm{~d}, J=4.58 \mathrm{~Hz}$, $3 \mathrm{H}), 7.94(\mathrm{~d}, J=4.71 \mathrm{~Hz}, 2 \mathrm{H}) .{ }^{13} \mathrm{C} \mathrm{NMR}\left(100 \mathrm{MHz}, \mathrm{CDCl}_{3}\right) \delta$, ppm 174.45, 167.71, 141.18, 140.67, 130.32, 127.84, 119.74, 117.89, 77.35, 77.03, 76.72, 44.54, 43.65, 36.13, 19.31.Anal. Calcd for: $\mathrm{C}_{20} \mathrm{H}_{22} \mathrm{~N}_{2} \mathrm{O}_{2} \mathrm{~S}$ : C, 62.16; H, 5.74; N, 7.25; O, 16.56; S, 8.30. Found: C, 62.15; H, 5.73; N, 7.24.

\section{N-(1-Isopropyl-2-oxoindolin-5-yl)-1-methyl-1H-pyrazole-5-carboxamide(6g)}

White solid; M.P. $180-181{ }^{\circ} \mathrm{C} ;{ }^{1} \mathrm{H}$ NMR (400 MHz, $\left.\mathrm{CDCl}_{3}\right) \delta$, ppm $1.46(\mathrm{~d}, J=10.88 \mathrm{~Hz}, 6 \mathrm{H}), 3.52$ (s, $2 \mathrm{H}), 4.21(\mathrm{~d}, J=8.67 \mathrm{~Hz}, 3 \mathrm{H}), 4.67-4.68(\mathrm{~m}, 1 \mathrm{H}), 6.66(\mathrm{~d}, J=1.80 \mathrm{~Hz}, 1 \mathrm{H}), 6.99(\mathrm{~d}, J=8.48 \mathrm{~Hz}, 1 \mathrm{H})$, 7.39-7.30 (m, 1H), $7.51(\mathrm{~d}, J=2.02 \mathrm{~Hz}, 1 \mathrm{H}), 7.60(\mathrm{~s}, 1 \mathrm{H}), 7.71(\mathrm{~s}, 1 \mathrm{H}) .{ }^{13} \mathrm{C}$ NMR $\left(100 \mathrm{MHz}, \mathrm{CDCl}_{3}\right) \delta$, ppm 174.5, 158.1, 140.7, 137.5, 135.2,131.8, 125.7, 120.2, 118.2, 109.8, 106.5, 43.6, 39.8, 36.0, 19.2.Anal. Calcdfor: $\mathrm{C}_{16} \mathrm{H}_{18} \mathrm{~N}_{4} \mathrm{O}_{2}$ : C, 64.41; H, 6.08; N, 18.78; O, 10.73. Found: C, 64.40; H, 6.07; N, 18.77.

\section{N-(1-Isopropyl-2-oxoindolin-5-yl)-4-methylisoxazole-3-carboxamide(6h)}

White solid; M.P. 190-191 ${ }^{\circ} \mathrm{C} ;{ }^{1} \mathrm{H}$ NMR $\left(400 \mathrm{MHz}, \mathrm{CDCl}_{3}\right) \delta$, ppm 1.47 (t, $\left.J=7.00 \mathrm{~Hz}, 6 \mathrm{H}\right), 2.56(\mathrm{~d}, J=$ $6.38 \mathrm{~Hz}, 3 \mathrm{H}), 3.51(\mathrm{~s}, 2 \mathrm{H}), 4.67-4.68(\mathrm{~m}, 1 \mathrm{H}), 6.99(\mathrm{t}, J=6.01 \mathrm{~Hz}, 1 \mathrm{H}), 7.30(\mathrm{dd}, J=8.47,1.72 \mathrm{~Hz}, 1 \mathrm{H})$, 7.61-7.44 (m, 2H), $8.80(\mathrm{~s}, 1 \mathrm{H})$. Anal.Calcd for: $\mathrm{C}_{16} \mathrm{H}_{17} \mathrm{~N}_{3} \mathrm{O}_{3}: \mathrm{C}, 64.20 ; \mathrm{H}, 5.72 ; \mathrm{N}, 14.04 ; \mathrm{O}, 16.04$. Found: C, 64.19; H, 5.71; N, 14.03. 


\section{BIOLOGICAL ASSAY \\ Bacterial and Fungal Strains}

The bacterial strains were purchased from the Public Health England Culture Collections. Fungal strains were collected from the department of biotechnology, Chaitanya P.G.College (autonomous), Hanamkonda-506001, Telangana, India. All microbial strains stored at $-80{ }^{\circ} \mathrm{C}$ were streaked on Luria-Bertani (LB) agar plate and incubated at $37^{\circ} \mathrm{C}$ for $24 \mathrm{~h}$. Fewer numbers of isolated colonies were chosen from each plate and suspended in $5 \mathrm{~mL}$ of LB broth in a hygienic culture vessel. The vessel was plugged with cotton and gestated with gentle shaking $(140 \mathrm{rpm})$ at $37^{\circ} \mathrm{C}$ for 20 hours.

\section{Preparation of Inoculums}

Following the protocol of the Kirby-Bauer disk diffusion assay, ${ }^{12}$ four to five well-isolated colonies of the same morphological type were picked with an inoculating loop, transferred into $5 \mathrm{~mL}$ of nutrient broth, and incubated at $37^{\circ} \mathrm{C}$ for $24 \mathrm{~h}$ until slight noticeable turbidity appeared. The turbidity of the vigorously growing broth cultures was then adjusted with broth to a density equivalent to that of a 0.5 McFarland standard, and the resulting suspensions were used as the initial inocula in the assay.

\section{Antimicrobial Assay}

The initial inocula of the test organisms, $100 \mu \mathrm{L}$, were swabbed over the surface of the agar media (20 $\mathrm{mL}$ ) in Petri dishes and let to be absorbed for $15 \mathrm{~min}$. Wells, $8 \mathrm{~mm}$ in diameter, were made with a sterile cork borer in the seeded agar plates. Solutions of the test compounds in DMSO $(100 \mu \mathrm{L} ; c 75$ and 100 $\mathrm{mg} / \mathrm{mL}$ ) were then loaded into the wells and incubated in the air at $37^{\circ} \mathrm{C}$ for $24 \mathrm{~h}$. The inhibition zone diameter was measured with a zone reader (Antibiotic Zone Scale). The positive control was the standard drug Gentamicin.

\section{RESULTS AND DISCUSSION}

As depicted in Scheme-1, new derivatives of indolin-2-ones $(\mathbf{6 a - h})$ were synthesized in five steps protocol. The key intermediate 5-amino-1-isopropylindolin-2-one $(\mathbf{4})^{13-15}$ was prepared from commercially available indoline-2,3-dioneand2-iodopropane in the presence of sodium hydride in DMF for reacting $10 \mathrm{~min}$ at room temperature to give 1-isopropylindoline-2,3-dione (1). Compound 1was reduced in the presence of hydrazine hydride at $130{ }^{\circ} \mathrm{C}$, yielded 1-isopropylindolin-2-one (2). ${ }^{12}$ Further, nitration of compound 2 was carried out by reacting with the nitrating mixture (sodium nitrate and Con. $\mathrm{H}_{2} \mathrm{SO}_{4}$ ) at room temperature for 30 min toyield1-isopropyl-5-nitroindolin-2-one $(3)^{13}$. The reduction of 1isopropyl-5-nitroindolin-2-one (3) in the presence of iron and $\mathrm{NH}_{4} \mathrm{Cl}$ in solvent mixture of ethanol and water (1:1) produced5-amino-1-isopropylindolin-2-one (4). ${ }^{14}$ Structure of the compound 4 was confirmed with the already reported melting point $453-455{ }^{\circ} \mathrm{C} .{ }^{14}$ As we found amine (4) compound as a better precursor, the same has been taken in to design amide compounds based on the acid-amine coupling reaction. Different acids (5a-h) in DMF solvent were used for the acid-amine coupling reaction with5amino-indolin-2-one (4)in the presence of EDC.HCl and HATU under MW irradiation to get compounds 6a-h in high yields. The conventional process to obtain the same compounds $\mathbf{6 a - h}$ produced low yields and the reaction time also more than under MW irradiation (Table-1). The structures of all the newly synthesized compounds were characterized by ${ }^{1} \mathrm{HNMR},{ }^{13} \mathrm{C}$ NMR spectral data and MASS.

Table-1: $N$-(1-Isopropyl-2-oxoindolin-5-yl) Substituted Amide Derivatives

\begin{tabular}{|c|c|c|c|c|c|}
\hline \multirow[t]{2}{*}{ Entry } & \multirow[t]{2}{*}{ Compound } & \multicolumn{2}{|c|}{$\begin{array}{c}\text { Room } \\
\text { Temperature }\end{array}$} & \multicolumn{2}{|c|}{ MW } \\
\hline & & Time & Yield $^{\mathrm{a}}$ & Time & Yield $^{\mathrm{a}}$ \\
\hline 1. & & $12 \mathrm{~h}$ & $45 \%$ & $10 \mathrm{~min}$ & $88 \%$ \\
\hline
\end{tabular}


RASĀYAN J. Chem.

Vol. 12 | No. 4 |1850 - 1856| October - December | 2019

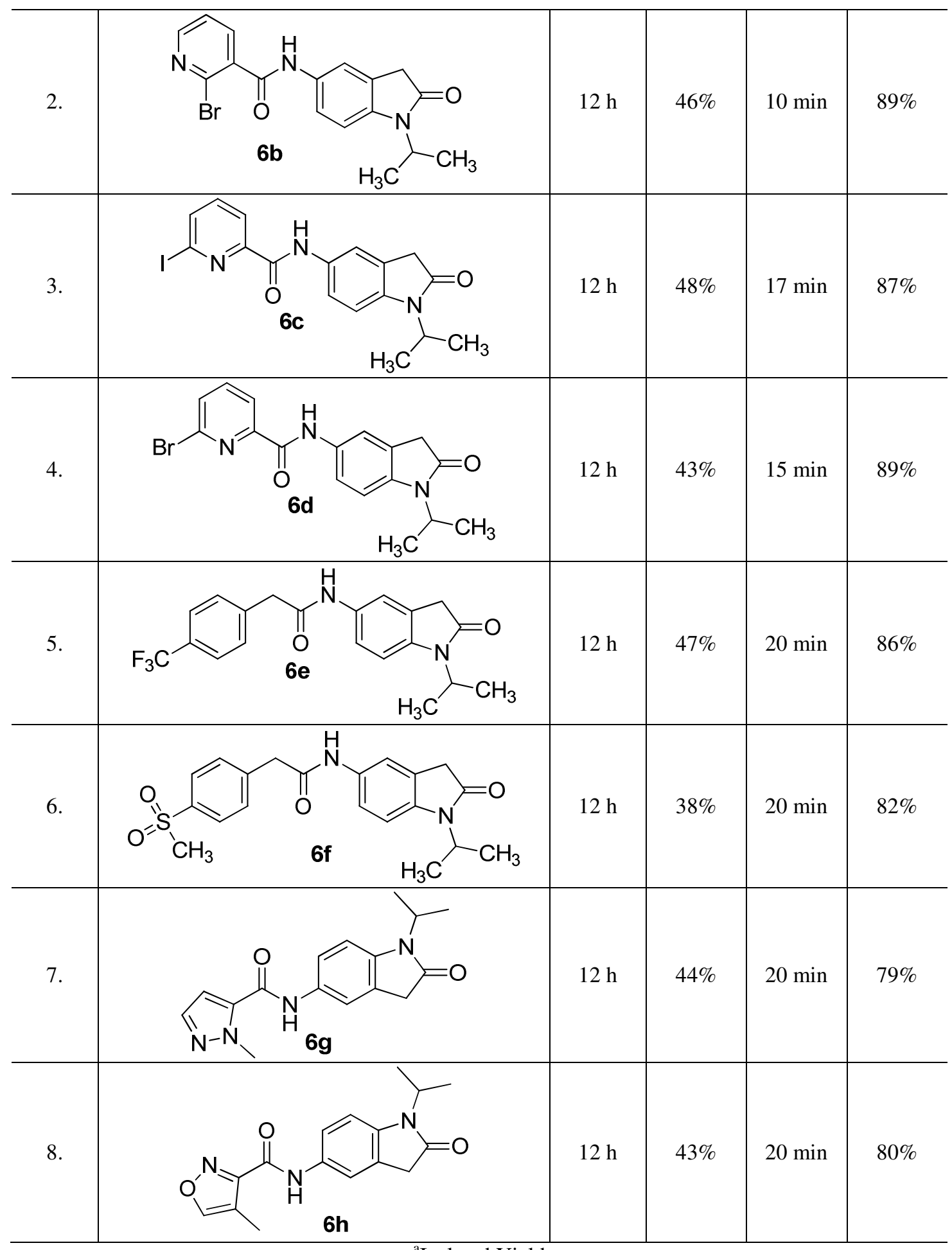

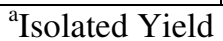

Table-2: In Vitro Antibacterial Activity of 6a-h

\begin{tabular}{l|c|c|c|c|c|c|c|c|c}
\hline \multirow{3}{*}{ Compound } & \multirow{8}{*}{$\begin{array}{c}\text { Conc. } \\
\mu \mathrm{g} / \mathrm{mL}\end{array}$} & \multicolumn{9}{|c}{ Gone of Inhibition (mm) } \\
\cline { 3 - 11 } & & M. $t u b$ & M. lut & B. sub & MRSA & P. aer & K. pne & $\begin{array}{c}\text { E. } \\
\text { coli }\end{array}$ & P. vul \\
\hline & 75 & 17 & 13 & 15 & 13 & 14 & 16 & 19 & 20 \\
\hline \multirow{2}{*}{$6 \mathrm{a}$} & 100 & 18 & 15 & 16 & 16 & 16 & 17 & 20 & 21 \\
\hline
\end{tabular}




\section{RASĀYAN J. Chem.}

Vol. 12 | No. 4 |1850 - 1856| October - December | 2019

\begin{tabular}{c|c|c|c|c|c|c|c|c|c}
\hline \multirow{2}{*}{$6 \mathrm{~b}$} & 75 & 21 & 19 & 19 & 18 & 19 & 19 & 23 & 24 \\
\cline { 2 - 10 } & 100 & 23 & 21 & 21 & 20 & 20 & 21 & 24 & 25 \\
\hline \multirow{2}{*}{$6 \mathrm{c}$} & 75 & 14 & 11 & 13 & 09 & 12 & 13 & 17 & 17 \\
\cline { 2 - 10 } & 100 & 16 & 13 & 13 & 10 & 12 & 14 & 18 & 19 \\
\hline \multirow{2}{*}{$6 \mathrm{~d}$} & 75 & 18 & 16 & 18 & 17 & 16 & 18 & 21 & 20 \\
\cline { 2 - 10 } & 100 & 20 & 17 & 20 & 19 & 17 & 19 & 22 & 23 \\
\hline \multirow{2}{*}{$6 \mathrm{e}$} & 75 & 25 & 23 & 25 & 24 & 23 & 21 & 26 & 25 \\
\cline { 2 - 10 } & 100 & 27 & 24 & 26 & 25 & 24 & 23 & 28 & 27 \\
\hline \multirow{2}{*}{$6 \mathrm{f}$} & 75 & 28 & 25 & 27 & 29 & 26 & 24 & 29 & 28 \\
\cline { 2 - 10 } & 100 & 32 & 26 & 29 & 30 & 27 & 26 & 31 & 29 \\
\hline \multirow{2}{*}{$6 \mathrm{~g}$} & 75 & 23 & 22 & 23 & 20 & 22 & 20 & 25 & 24 \\
\hline \multirow{2}{*}{$6 \mathrm{~h}$} & 100 & 25 & 24 & 26 & 23 & 23 & 22 & 28 & 24 \\
\hline \multirow{2}{*}{ Zentamicin } & 75 & 27 & 23 & 26 & 27 & 24 & 23 & 27 & 27 \\
\cline { 2 - 10 } & 100 & 30 & 25 & 28 & 28 & 25 & 25 & 29 & 28 \\
\cline { 2 - 10 } & 100 & 32 & 30 & 33 & 33 & 31 & 30 & 33 & 31 \\
\hline
\end{tabular}

Mycobacterium tuberculosis (M. tub), Micrococcus luteus (M. lut), Bacillus subtilis (B. sub), Methicillin-resistant Staphylococcus aureus (MRSA), Pseudomonas aeruginosa (P. aer), Klebsiella pneumonia (K. pne), Escherichia coli (E. coli), Proteus vulgaris (P. vul), No Activity (NA).

Table-3: In Vitro Antifungal Activity of 6a-h

\begin{tabular}{|c|c|c|c|c|}
\hline \multirow{2}{*}{ Compound } & \multirow{2}{*}{$\begin{array}{l}\text { Conc. } \\
\mu \mathrm{g} / \mathrm{mL}\end{array}$} & \multicolumn{3}{|c|}{ Zone of Inhibition (mm) } \\
\hline & & M. canis & E. floccosum & M. gypseum \\
\hline \multirow{2}{*}{$6 a$} & 75 & 16 & 15 & 17 \\
\hline & 100 & 18 & 16 & 19 \\
\hline \multirow{2}{*}{$6 \mathrm{~b}$} & 75 & 17 & 15 & 18 \\
\hline & 100 & 21 & 17 & 19 \\
\hline \multirow{2}{*}{$6 \mathrm{c}$} & 75 & 14 & 13 & 16 \\
\hline & 100 & 15 & 14 & 16 \\
\hline \multirow{2}{*}{$6 \mathrm{~d}$} & 75 & 15 & 14 & 16 \\
\hline & 100 & 17 & 15 & 17 \\
\hline \multirow{2}{*}{$6 \mathrm{e}$} & 75 & 20 & 18 & 19 \\
\hline & 100 & 23 & 19 & 19 \\
\hline \multirow{2}{*}{$6 \mathrm{f}$} & 75 & 23 & 19 & 18 \\
\hline & 100 & 25 & 20 & 19 \\
\hline \multirow{2}{*}{$6 \mathrm{~g}$} & 75 & 19 & 17 & 18 \\
\hline & 100 & 22 & 18 & 20 \\
\hline \multirow{2}{*}{$6 \mathrm{~h}$} & 75 & 22 & 18 & 19 \\
\hline & 100 & 25 & 19 & 20 \\
\hline \multirow{2}{*}{ Nystatin } & 75 & 25 & 20 & 19 \\
\hline & 100 & 28 & 23 & 24 \\
\hline
\end{tabular}

Microsporum canis (M. canis), Epidermophyton floccosum (E. floccosum), Microsporum gypseum (M. gypseum).

All the final target compounds $(\mathbf{6 a - h})$ were screened for their antibacterial activity against four Grampositive and four-gram negative bacterial species and the analogs $\mathbf{6 a - h}$ showed pronounced antibacterial activity compared to standard drug Gentamycin (Table-2).Among all the tested compounds $\mathbf{6 f}, \mathbf{6 h}, \mathbf{6 e}, \mathbf{6 g}$ and $\mathbf{6 b s h o w e d ~ p r o m i s i n g ~ z o n e ~ o f ~ i n h i b i t i o n ~ c o m p a r e d ~ t o ~ a l l ~ o t h e r ~ c o m p o u n d s . ~ T h e s e ~ c o m p o u n d s ~ ( 6 a - h ) ~}$ were also assessed for their antifungal activity against three fungal species and the compounds $6 \mathbf{6}, \mathbf{6 h}, \mathbf{6 e}$ and $\mathbf{6 b}$ exhibited significant antifungal activity compared to standard drug Nystatin (Table-3).

\section{CONCLUSION}

A series of novel $N$-(1-isopropyl-2-oxoindolin-5-yl) substituted amide derivatives $6 \mathbf{6}-\mathbf{h}$ were synthesized using Microwave irradiation with short time of process and in high yields. All target compounds were characterized by spectral dat. Final derivatives $(\mathbf{6 a}-\mathbf{h})$ were screened for their antimicrobial activity and 


\section{RASĀYAN J. Chem.}

Vol. 12 | No. 4 |1850 - 1856| October - December | 2019

demonstrated moderate to good activity. Among all the compounds, $6 \mathbf{f}, \mathbf{6 h}, \mathbf{6 e}, \mathbf{6 g}$ and $\mathbf{6 b w e r e}$ found potent antimicrobial activities at concentrations 75 and $100 \mu \mathrm{g} / \mathrm{mL}$. These results positively encouraged us to further developing novel bioactive agents.

\section{ACKNOWLEDGMENTS}

P. Shankaraiah and A Kishore Kumar are thankful to Vardhaman College of Engineering, Shamshabad, Hyderabad, Telangana.

\section{REFERENCES}

1. O. Marko, S. G. Grdadolnik, M. Kotnik, R. Jerala, M. Filipic and T. Solmajer, Bioorg. Med. Chem. Lett., 15, 5207(2005), DOI:10.1016/j.bmcl.2005.08.068

2. L. Sun, N. Tran, F. Tang, H. App, P. Hirth, G. McMahon, and C. Tang, J. Med. Chem., 41, 2588(1998), DOI:10.1021/jm980123i

3. M. Sassatelli, E. Debiton, B. Aboab, M. Prudhomme and Moreau, Eur. J. Med. Chem.,41, 709(2006), DOI:10.1016/j.ejmech.2006.03.021

4. J. Honghe, Y. Zhu, Y. Shao, J. Liu, Y. Yuan and X. Jia, Bioorganic Med. Chem. Lett., 20, 7349(2010), DOI:10.1016/j.bmcl.2010.10.056

5. F. Bouchikhi, E. Rossignol, M. Sancelme, B. Aboab, F. Anizon, D. Fabbro, M. Prudhomme and P. Moreau, Eur. J. med. Chem., 43, 2316(2008), DOI:10.1016/j.ejmech.2008.01.010

6. D. Harish and A. Kumar Madan, J. Mol. Model., 11, 525(2005), DOI:10.1007/s00894-005-0276-3

7. T.I. El-Emary, R.A. Ahmed and E.A. Bakhite, J. Chin. Chem. Soc., 48, 921(2001), DOI: $10.1002 /$ jecs.200100134

8. A. Jarrahpour, D. Khalili, E. De Clercq, C. Salmi and J.M. Brunel, Molecules, 12, 1720(2007), DOI: $10.3390 / 12081720$

9. M. Thankamony and K. Mohanan, Asian J. Chem., 19, 2877(2007).

10. A. AH, J. Chin.Chem Soc., 53, 663(2006), DOI:10.1002/jccs.200600087

11. A. Dongamanti, V.H. Rao and S. Peddolla, Heterocycl Commun., 19, 363(2013), DOI:10.1515/hc2013-0046

12. P. Shankaraiah, S. Veeresham, and A.K.D. Bhavani, Russ. J. Gen. Chem., 86, 368(2016), DOI:10.1134/S1070363216020286

13. G. Mikhail, G. Sumithra, V.N.J. Vara Prasad and W. L. Gary, WO 2007091147, PCT Int. Appl., 752, 000284(2006).

14. W. L.Gary, J. Rama, R. R. Adam, V.N. J.Vara Prasad, F. G. Mikhail, WO 2006106426, PCT Int. Appl., 978, 002712 (2006).

15. X. Xiu-Hua, X. Wang, G. Liu, E. Tokunaga and N. Shibata, Org. Lett.,14, 2544(2012), DOI: $10.1021 / \mathrm{ol} 300842 \mathrm{~d}$

[RJC-5445/2019] 